respects, similar, I do not feel able to make a positive statement in regard to the diagnostic value of this test in all cases. However, the difference in the appearance of the crystals, so far as I have yet observed, seems to be sufficiently great to serve as a means of distinguishing butter from oleomargarine.

From oleomargarine I have obtained perfect crystals of lard and of beef fat. Butter crystals are somewhat lard-like in appearance, but are much shorter and are frequently in rosette clusters.

The refractometer readings given in the table were made with a Zeiss butyrorefractometer. The first column gives the scale reading of the original sample, the second, or part soluble in the fusel oil at $17^{\circ} \mathrm{C}$., and the third column, of the insoluble portion. The indices of refraction corresponding to the scale readings of this instrument can be found in Wiley's Agricultural Chemistry.

The fusel oil used in this work had a boiling-point of $129^{\circ} \mathrm{C}$. and specific gravity of 0.8159 .

\title{
CONTRIBUTIONS TO THE CHEMISTRY OF DIDYMIUM.
}

\section{BY I. M. DENNIS AND E. M. Chamot.}

Recelved August 28,2897

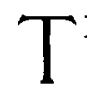

$\mathrm{HE}$ announcement by Mosander ${ }^{1}$ in 1842 of his discovery of a new element which he found accompanying cerium and lanthanum, and to which he gave the name didymium, was followed by careful and able investigations by Mosander and others upon the chemistry of this obscure group. The information which they obtained by means of purely chemical methods was, however, both meager and contradictory, ${ }^{2}$ and it was not until the discovery by Gladstone ${ }^{3}$ had put into chemists' hands a new analytical method that the results began to be fairly definite and satisfactory.

The spectroscope affords a means of detecting small amounts of earths yielding absorption spectra, and thus makes possible the preparation of purer compounds of such elements as cerium, lanthanum and yttrium, which give no absorption bands; but the most interesting results of the employment of spectroscopic

1 Ann. Chem. Pharm., 44, 125.

2 Hermann: J.prakt. Chem., 34, 182, doubts the existence of didymium.

3 "On an Optical Test for Didy mium," J. Chem. Soc., Io, 219. 
methods are seen in the gradual resolution of Mosander's didymium into a number of component earths. Gladstone' mentioned only two bands in the absorption spectrum of a didymium solution, while Erdmann ${ }^{2}$ mapped seven lines, and said that there might be an eighth in the extreme violet. Rood ${ }^{3}$ stated that there are twelve, and Bahr and Bunsen ${ }^{4}$ gave measurements of eleven, while later observers ${ }^{5}$ describe about twenty bands. These twenty bands which, ten years ago, Krüss and Nilson ascribed to didymium, show that the separation of old didymium into several elements had already begun, for they do not include several bands which had formerly been thought to be due to didymium, but which Boisbaudran in 1878 had shown to be caused by a new element which he had separated and which he named samarium. In I885 Auer von Welsbach ${ }^{7}$ succeeded in separating didymium into two earths, which he called neodym and praseodym, but which, following the example of Kriiss, ${ }^{8}$ should be termed neodidym and praseodidym. These two earths have markedly different properties, the neodidymium giving rose red salts, while those of the praseodidymium are green. There can be little doubt, however, that both are composite and that neither one is an element in the present acceptation of that term. Krïss and Nilson, ${ }^{9}$ upon examining didymium extracted from many different minerals, found that the same absorption bands varied in relative intensities in the samples of didymium from these various sources, and concluded that our present didymium probably consists of not less than nine elements. Crookes has made the suggestion that each element may yield but one absorption band, and says: "According to this hypothesis, therefore, neodymium and praseodymium must not be considered as actual chemical elements, but only the names given to two groups of molecules into which the complex molecule didym-

1 Loc. cit.

2 J.prakt, Chem., 85, 394 .

3 Chem. News, 6, 140.

4 Zischr, anal, Chem., 5, Iog.

5 Lecoq de Boisbaudran: Spectres lumineux, Texte, p. 87; Krüss and Nilson: Ber, $d$. chem. Ges, $20,2 \mathbf{I}^{6}$.

6 Compt. rend., 89, $2 \mathrm{I} 2$.

7 Monatshefte, 6,477 .

8 Ann. Chem. (Liebig), 265, 3. Krüss and Nilson ask (Ber. d. chem, Ges., 20, 2:56), "Was bedeutet 'dym'?"

9 Ber.d. chem. Ges., 20, 2166. 
ium splits up by one particular method of fractionation." ' Thompson ${ }^{2}$ thinks that the six bands in the blue and violet, having approximately the wave-lengths $482,476,469,462,444$ and 428 , are due to at least five different components. Becquerel and others similarly state their belief in the compound nature of didymium.

It would seem natural, then, that having become convinced of the compound character of old didymium, chemists should at once undertake the isolation of the various constituents of this earth. Many of our ablest investigators have endeavored to do this, but the various bases are so closely allied in their chemical characteristics that, up to the present time, only approximate separations have been effected, and these separations have, for the most part, been accomplished by the employment of methods of fractionation that constant use has shown to be capable of giving only imperfect separations, and that at the expenditure of much time and labor. The need, then, at the present time is for new methods of separation or critical examination of the older methods, so that we may be able to obtain sharper results in shorter time. The experimental work described below was undertaken with this end in view, and while we are as yet unable to offer new data concerning the components of didymium, we trust that our observations upon the splitting of didyminm may be of interest to workers in this field.

The mixture of earths, which was used for the work, was extracted from monazite sand from Brazil. The sand was decomposed by sulphuric acid and the rare earths were separated from the other elements present by the usual methods. The large amount of cerium which accompanied the other earths was removed by the chlorine method devised by Mosander, as this was found to be more suitable to the treatment of large amounts (we had in hand 9,200 grams of the dry oxalates) than was the fusion of the nitrates with a mixture of sodium and potassium nitrates. ${ }^{3}$

The following procedure was finally adopted: Tall bottles of a capacity of from fifteen to twenty liters, were fitted with twohole rubber stoppers, one hole carrying a glass tube of about

1 Chem. News, 54, 27.

2 Chem. News, 55, 227.

3 Dennis and Magee: This Journal, 16, 649. 
one $\mathrm{cm}$. diameter, reaching to the bottom of the bottle, the other an exit tube ending just below the stopper. The bottles were then connected in series by rubber tubing, so that the chlorine would pass through all of them in turn. Eight to ten liters of the moderately dilute solution of nitrates of the earths was poured into each bottle and a strong solution of potassinm hydroxide was then added until the reaction was distinctly alkaline. Each bottle was filled with water to within six inches of the top. Chlorine gas was then led into the first bottle at a rapid rate, any unabsorbed gas passing into the second. By shaking the first bottle from time to time, it was found easy to determine when no further absorption of gas was there taking place. The treatment was then continued a little longer, when the first bottle was removed and the gas passed directly into bottle number two, a fresh bottle being placed at the end of the row. By operating in this way there were always three bottles in line, the last being a fresh one. No loss of chlorine could occur and the operation being continuous, a large amount of material could be treated in a short space of time. After allowing the chlorinated portions to stand until the insoluble ceric hydroxide had settled, the supernatant solution was siphoned off and filtered. The clear solution thus obtained contained the earths of the didymium and yttrium groups free from ceria. This solution was transferred to tall glass cylinders of about twenty liters capacity and the liquid was violently mixed by means of the air blast or a Rabe turbine, oxalic acid, meanwhile, being slowly run in until in excess. The precipitate was allowed to settle, the supernatant liquid was drawn off with a siphon and the cylinder was again filled with distilled water. After each addition of water the contents of the cylinder was stirred for an hour, and the above operation was repeated until the wash-water was free from chlorides. More didymium was separated from the insoluble ceric hydroxide by repeatedly washing the latter with water and then dissolving it in hydrochloric acid, adding potassium hydroxide and chlorinating once more. The chlorine which was used came from a cylinder containing ninety pounds of the liquefied gas. This was found to be cheaper and easier to control than the production of the gas by the usual methods.

The thoroughly washed oxalates of the earths freed from 
cerium as above, were dried in an air-bath, ignited in large platinum dishes and dissolved in dilute nitric acid. The clear pink solution showed no trace of cerium when tested with hydrogen peroxide and ammonium hydroxide, and was therefore ready for fractionation, unless it was desired to first remove the earths belonging to the yttrium group. To remove the yttrium group, the solution of the nitrates of the earths was diluted and placed in a tall cylinder whose contents could be rapidly stirred from top to bottom by means of a turbine. Potassium sulphate was added in sufficient quantity to constantly maintain quite a thick layer of the crystais on the bottom. In this way a very satisfactory separation can be accomplished in a far shorter time than by any method with which we are familiar. In the absence of a turbine, almost as good results can be obtained by using a strong air blast, but in this case a quantity of potassium sulphate should be maintained near the surface of the liquid by means of a percolator or similar device.

The precipitate from the above treatment contained the double sulphates of the didymium group. It was washed repeatedly with a saturated solution of potassium sulphate and was finally thrown upon a Witt plate and sucked dry with the suctionpump. The insoluble sulphates were then dissolved in a moderately dilute ammonium acetate solution, ' filtered, and the clear liquid precipitated with ammonium hydroxide, hydrochloric acid sufficient to decompose the ammonium acetate having first been added. The hydroxides were washed as usual until no further test for sulphate could be obtained by barium chloride. These hydroxides were then dissolved in nitric acid and this solution was used in the subsequent work. In one case in which the yttrium group was removed after a series of fractional crystallizations had been completed, it was found that the double potassium sulphate salts of the earths at the neodidymium end of the series dissolved much more easily in ammonium acetate than did the praseodidymium fractions.

The method of fractionation which was first tried was that of Welsbach, ${ }^{2}$ but the details which he gives, especially concerning the strength of acid and the amount of water to be added, are

1 Urbain: Bull. Soc. Chim., I5, 349, 2896.

2 Monatshefte, 5,477 . 
vague and our results when following his directions as closely as possible, were quite unsatisfactory.

It was soon found that the results of the fractionation were greatly influenced by the amount of the nitric acid present. If a large amount of nitric acid is added, the lanthanum double salt separates in the first two or three fractions, and the subsequent splitting of the didymium is then very slow; if, on the other hand, the acid be too weak, a long series of fractions which show but slight differences, is obtained.

The method used by Bettendorff ${ }^{1}$ with material high in lanthanum and low in didymium was next tried, but as our oxides contained didymium in excess, the results were again unsatisfactory. It was found, however, that although Bettendorff's method did not produce much effect on the didymium, it appeared to rapidly remove samarium, the absorption bands of this earth being quickly eliminated, whereas the didymium bands showed but little change.

While working upon Bettendorff's scheme of separation, a series of fractions prepared by Welsbach's method had been allowed to stand undisturbed for several days. Uponexamining the middle members of this series it was noticed that where the solution had crawled up on the sides of the dishes and crystallized, the crust thus formed was of a deep rose-red color, while the crystals which had formed in the solution were of an entirely different shade of pink. The two kinds of crystals were carefully separated and dissolved in water. The absorption spectra of the two solutions showed that a marked splitting of didymium had taken place, the crystalline crusts which had formed on the sides of the dishes giving a spectrum in which the neodidymium bands were much stronger than those assigned to praseodidymium; there was also a remarkable increase in the intensity of the samarium bands. An aqueous solution of some of the other crystals showed a corresponding increase in the intensity of the praseodidymium bands.

These observations naturally led to the idea that the splitting of didymium might be more rapidly attained by abandoning the customary procedure of concentration by heat and rapid crystallization, and subjecting the solutions to spontaneous evaporation

1 Ann. Chem. (Liebig), 256, I63. 
and slow crystallization. The results exceeded our expectation, for upon making a series of fractions in this manner, very marked spectrum changes were speedily obtained. The sharpness of the separation by slow crystallization is illustrated by the two absorption spectra shown in Plate II. Only the lines in the blue and violet are here mapped, since the changes are greatest in that portion of the field. A portion of the solution containing the double ammonium nitrate was allowed to stand for five days and slowly crystallize. The mother-liquor was then poured off. Its spectrum (II, $a$ ) shows very strong neodidymium bands, and praseodidymium bands which are relatively much weaker. A saturated solution of the crystals gave the spectrum shown in II, $b$, in which the neodidymium bands are much weaker than those of praseodidymium. The thickness of the solution in each case was ten $\mathrm{mm}$. To test more thoroughly the efficiency of this slow crystallization, somewhat over one kilo of oxides of monazite earths, free from ceria, was converted into double ammonium nitrates, a small amount of nitric acid added, and the whole evaporated to such a concentration that partial crystallization took place only after forty-eight hours. The motherliquor was then poured off and set aside to crystallize spontaneously. The crystals were dissolved in dilute nitric acid by the aid of heat, and allowed to stand. The crystals and motherliquor thus obtained were treated as before. After about twenty such fractions had been formed, all those of about the same color, shade, and intensity were united and each portion thus formed was then fractioned separately, similar fractions being from time to time united so as to form a long unbroken series. This process was continued until a series of 105 fractions of the double nitrates had been obtained.

It was found that while the greater part of the lanthanum separated in the first few portions, much of it, contrary to expectation, was carried along to the latter fractions, depositing gradually with the other earths until apparently a certain relative concentration of the different earths was reached, when white crystals again separated, the subsequent fractions becoming more and more intensely pink, until finally the beautiful rose-red salts of the neodidymium and samarium earths were formed. Then suddenly light-colored crystals would appear in the suc- 
ceeding fractions, and there would again be seen the same series of color changes as had before been observed. For example, fractions $I$ to Io were almost pure lanthanum ammonium nitrate, I I to I6 showed a growing intensity of the characteristic green tint of the praseodidymium components, 17 was whitish with a tinge of brownish pink, which increased in intensity to 24,25 had the same tint as 17 , but the succeeding fractions up to 29 were decidedly pink, the color growing rapidly darker; 30 showed another falling off to a light color, as did $38,48,57,7^{2}$, 78,80 , and 88 . Above 88 the pink color increased to the end of the series. Throughout the whole series, the spectra of the light-colored crystals, no matter in what portion of the series. these lighter crystals had appeared, showed the bands of praseodidymium much more strongly than those of the neodidymium earths, and the samarium bands were generally almost invisible.

Determinations, by the method of Gibbs, of the atomic nass of the earths in the almost colorless fractions gave on the average $\dddot{R}=$ about 139 . Assuming then that these light-colored fractions consisted largely of lanthanum, an examination of the series disclosed the fact that the most marked splitting of didymium was noticeable in fractions but little removed from the light-colored portions, and that the amount of free nitric acid present exerted a powerful influence upon the character of the crystals. It seemed apparent, also, that slow crystallization had been much more effective than the rapid process hitherto employed.

Further experimentation led to the adoption of the following method, which has yielded, on the material from Brazilian monazite, most excellent results :

To a moderately concentrated solution of nitrates of the rare earths, free from ceria and high in lanthana, ammonium nitrate is added in the proportion of four molecules of $\mathrm{NH}_{4} \mathrm{NO}_{3}$ to one of $\mathrm{R}_{2} \mathrm{O}_{3}$ where $\dddot{\mathrm{R}}$ can be assumed to have a mean atomic mass of 142. Sufficient nitric acid (sp. gr. I.42) is then added to the solution so as to have one part of acid to four parts of water, by volume. The solution, after thorough stirring, is then evaporated to such a point that nearly all the salts will crystallize out in from twenty-four to thirty-six hours. The small amount of 


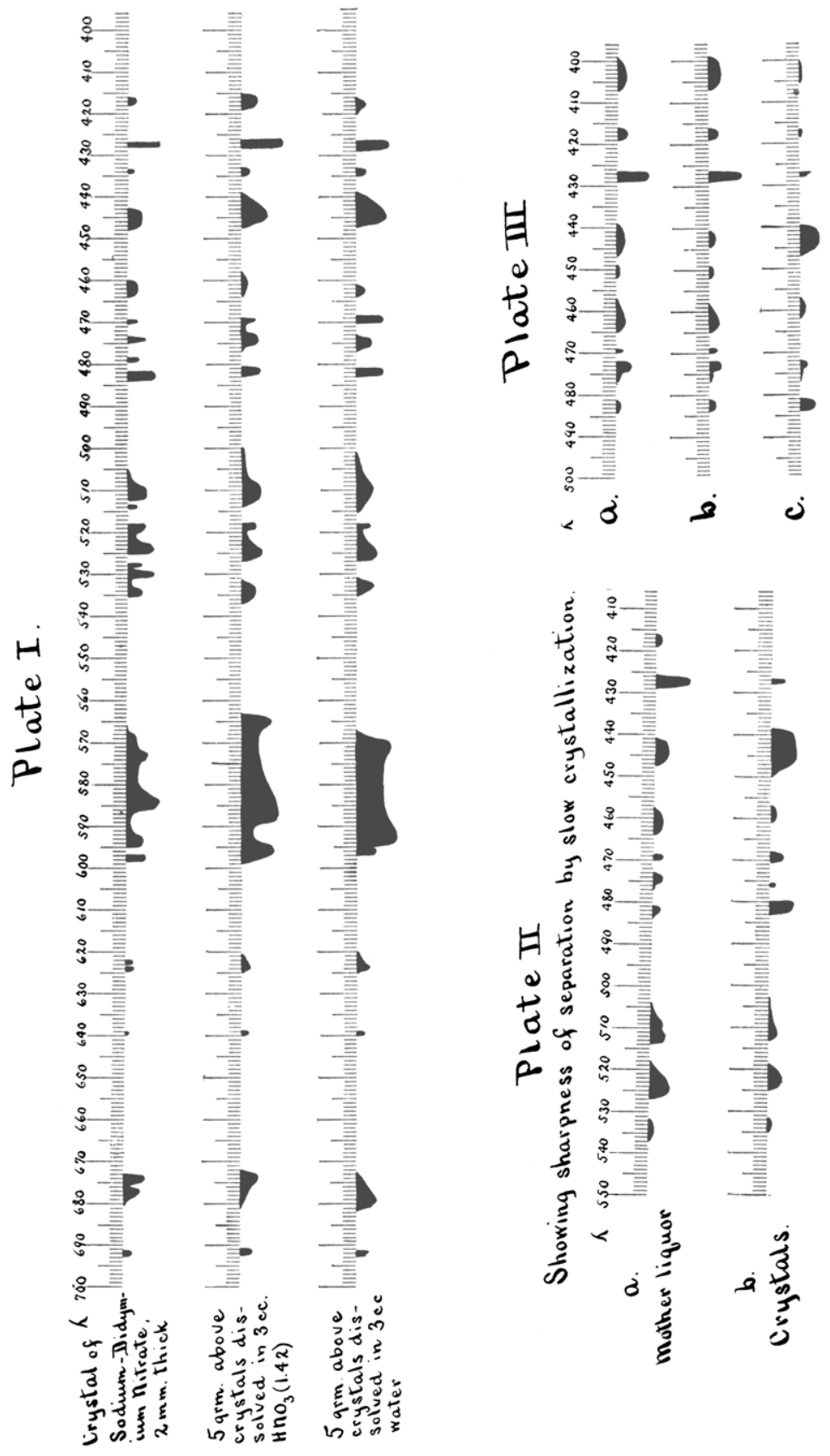



mother-liquor is then set aside and allowed to stand undisturbed until a good crop of crystals has formed; the mother-liquor from this fraction is poured off and allowed to crystallize spontaneously as before. The large crop of crystals from the original solution is dissolved with the aid of gentle heat, in such an amount of moderately dilute nitric acid ( $I: 4)$ that crystals begin to form in from five to ten hours. After a large proportion of the salts of the earths has separated, the mother-liquor is poured off and allowed to crystallize spontaneously. The crystals are dissolved as before in $\mathbf{I}: 4$ nitric acid. The same treatment is given to all crystals and mother-liquors obtained. From time to time such crystals and mother-liquors, irrespective of their position in the series, whose absorption spectra are approximately similar and whose colors are alike, are united and serve as the starting-point of new series of fractions, which in turn are similarly united. The absorption spectra of the different fractions should be constantly compared and like fractions united, since the rapidity of the method will depend very largely upon the judgment of the operator in properly uniting the various members of the series. The absorption spectra of the different fractions can easily be observed by reflected light, as they lie in the dishes, it being simply necessary to direct a pocket spectroscope toward them. It should be remembered, however, in comparing these spectra, that the spectrum of a crystal is generally very different from that of its solution. ${ }^{1}$

It is seldon, if ever, necessary to remove the crystals from the dishes in order to sufficiently free them from the mother-liquor; they form compact masses, and the supernatant solution can be thorougialy removed by simply tipping the evaporating dishes and leaving them in that position for a short time.

As the neodidymium end of the series is approached, lanthanum ammonium nitrate which has been obtained in the first fractions, is added to the fractions, together with a little nitric acid ( $1: 4$ ), and the whole is gently heated, thoroughly stirred, and set aside to slowly crystallize. The amount of lanthanum

1 Several years ago Lawrence Smith and I.ecoq de Boisbaudran (Compt. rend., 88, II67) called attention to the change which is caused in the absorption spectrum of didymium when vitric acid is added to the aqueous solution. Becquerel has described (Compt.rend., 104, 165 ) the variations in the spectra of the crystalline compounds of didymium. In further illustration of these peculiar phonomena. we have ventured to present the three spectra as shown in Plate $I$. 
salts to be added will, of course, depend upon the volume of the solution and the proportion of didymium earths present.

The above method possesses one serious drawback. The double ammonium nitrates of the didymium earths are extremely deliquescent, and in a moist atmosphere their solutions refuse to crystallize. By carrying on the work during the winter months in dry, steam-heated rooms, however, no difficulty whatever was experienced.

When the work was conducted in a fairly dry atmosphere it was found that more satisfactory results could be obtained by adhering to the use of ammonium nitrate than by changing, for the last series of fractions, to sodium nitrate, as Welsbach suggests. For example, it was found possible to obtain in one month, from 800 grams of the mixed oxides, a sample of praseodidymium, which in a saturated solution ten $\mathrm{cm}$. thick, showed only the faintest traces of the bands ascribed to neodidymium.

The fact that the presence of lanthanum facilitates the separation of these two components of didymium was mentioned by both Welsbach and Bettendorff. Schottländer appears to be the only one who added the lanthanum salt to some of his later fractions, but he seems to have discontinued this addition after a few series of fractions had been prepared.'

In our work it was found that whenever lanthanum had been largely removed from the solutions under treatment, crystallization took place only with great difficulty and such solutions, when they did crystallize, gave fractions that differed but little from one another in their absorption. spectra. If, however, all of these same fractions were again united and a small amount of lanthanum ammonium nitrate added, an immediate change took place in the absorption bands of the different fractions then obtained. Earths high in praseodidymium separated in the early fractions while the last fractions gave only faint bands of this component. The greater part of the samarium seemed to remain in the last mother-liquor. The results of one such crystallization after the addition of the lanthanum salts are shown in Plate III, $a, b, c, a$ shows the spectrum of the fraction before the addition of lanthanum. One hundred and twenty grams of lanthanum ammonium nitrate was then added

1 Ber, d. chem. Ges, 25, 392. 
and the solution allowed to slowly crystallize. $b$ gives the spectrum of the mother-liquor from the resulting crystals, showing increase in intensity of the neodidymium and samarium bands; $c$ is the spectrum of the solution of the crystals, showing marked weakening of the neodidymium bands, and strengthening of the praseodidymium.

These changes in the absorption bands cannot be due merely to the presence of lanthanum in the solutions, since the effect of such addition was repeatedly tried on different solutions and no changes in the character of the absorption bands could be detected other than a uniform weakening due to dilution. As soon, however, as crystallization began, the crystals and motherliquor showed differences from the original solution.

In fractioning by this method, it was noticed that the heavy band in the orange was not resolved into the component bands described by Welsbach, even when the remainder of the lines had undergone marked changes, this observation confirming the statement of Crookes that " probably didymium will be found to split up in more than one direction, according to the method adopted." In certain fractions the relative intensities of the bands bore out the observations of previous investigators as to the compound nature of both neodidymium and praseodidymium. Thus in one portion the neodidymium band $\lambda-428$ was intense, while the other didymium bands were comparatively faint. We also obtained fractions which showed the band $\lambda-443$ much more strongly than any other bands.

Corneli University, August, I897.

\section{THE TITRATION OF STANNOUS SALTS WITH IODINE.}

BY S. W. YOUNG.

Received July 3r, 8897 .

RESENIUS ${ }^{3}$ describes very briefly a method by Lenssen, for titrating stannous chloride with iodine in nentral or faintly alkaline solution. As was pointed out in a previous paper, ${ }^{4}$ it was evident that the action of iodine on stannous chloride was a quantitative one also in acid solution. Further study has con-

1 Chem. News, 54, 27.

2 See also Crookes: $J$. Chem. Soc., 56, 260.

3 syst. Quant. Chem. Anal., Ist Am. Ed., p. 342 .

4 This Journal, 29,517 . 